\title{
Vitamin B12 Supplementation in Treating Major Depressive Disorder: A Randomized Controlled Trial
}

\author{
Ehsan Ullah Syed ${ }^{2}$, Mohammad Wasay ${ }^{1, *}$ and Safia Awan ${ }^{1, *}$ \\ ${ }^{1}$ Department of Psychiatry and Medicine (Neurology), Aga Khan University, Karachi, Pakistan \\ ${ }^{2}$ Department of Psychiatry Penn State Milton S Hershey Medical Center Penn State College of Medicine, Hershey, PA, \\ USA
}

\begin{abstract}
Background/Objective: Recent literature has identified links between vitamin B12 deficiency and depression.We compared the clinical response of SSRI-monotherapy with that of B12-augmentation in a sample of depressed patients with low normal B12 levels who responded inadequately to the first trial with the SSRIs.

Methods: Patients with depression and low normal B12 levels were randomized to a control arm (antidepressant only) or treatment arm (antidepressants and injectable vitamin B12 supplementation).

Results: A total of 199 depressed patients were screened. Out of 73 patients with low normal B12 levels 34 (47\%) were randomized to the treatment group while 39 (53\%) were randomized to the control arm. At three months follow up $100 \%$ of the treatment group showed at least a $20 \%$ reduction in HAM-D score, while only $69 \%$ in the control arm showed at least a $20 \%$ reduction in HAM-D score $(\mathrm{p}<0.001)$. The findings remained significant after adjusting for baseline HAM-D score $(\mathrm{p}=0.001)$.
\end{abstract}

Conclusion: Vitamin B12 supplementation with antidepressants significantly improved depressive symptoms in our cohort.

Keywords: Depression, vitamin B12, antidepressants, RCT.

\section{INTRODUCTION}

Major Depressive Disorder is an important global public health problem associated with the significant burden and is projected to be the second leading cause of disability by 2020 [1]. Prevalence figures worldwide range between $4.2-$ $17 \%$ and a systematic review of studies from Pakistan gave the estimates as high as $34 \%$ for anxiety and depressive disorders [2]. Clinical guidelines recommend the use of SSRIs as first-line treatment [3]. Remission rates in the acute phase of treatment are $30-40 \%$ and an overall $30 \%$ show poor response to anti-depressant monotherapy [4-6]. In such cases, addressing other co-morbid conditions is suggested in addition to upgrading or augmenting anti-depressant doses [3].

Pakistan, a developing country of 17 million people, is fraught with poverty, inadequate nutrition and underprivileged health systems. Several small-case studies from Pakistan show vitamin deficiencies to be common $[7,8]$. A small study from Islamabad on a clinical population of patients with megaloblastic anemia reported figures of $76 \%$ with vitamin B12 deficiency [9]. Although no large scale studies on general population are available here, studies from neigh-

*Address correspondence to these authors at the Department of Medicine/ Neurology, The Aga Khan University, Stadium Road, Karachi 74800, Pakistan; Tel: (9221) 4930051; Ext: 4665, 4681; Fax: (9221) 4934294;

E-mail: mohammad.wasay@aku.edu; mohammadwasay@hotmail.com boring countries that share the culture and climate show vitamin deficiencies to be common [10-12]. A study on a healthy Indian population showed the prevalence of B12 deficiency to be $47 \%$ [13].

Vitamin B12 plays an important role in DNA synthesis and neurological function. Its deficiency is associated with hematological, neurological and psychiatric manifestations of which the latter includes irritability, personality change, depression, dementia and rarely, psychosis [14]. Recent literature has identified the links between this vitamin deficiency and depression. High B12 levels in serum are associated with good treatment response, high homocysteine levels which are common in folate / B12 deficiency and in those suffering from depression are associated with poor response to anti depressant treatment [15-17]. Hyperhomocysteinemia may have direct effects on neurotransmitters implicated in depression [18].

Randomized trials have shown that folate and other nutritional supplementations have a significant effect in treating the treatment-resistant depression [19,20]. Folate deficiency has also been linked with the delay in treatment response as well as relapse $[21,22]$.

To date no adequately designed trial compares antidepressant monotherapy with B12 augmentation. We aimed to compare the clinical response of antidepressants mono- 
therapy with that of B12-augmentation in a sample of depressed patients with low normalB12 levels $(190 \mathrm{pg} / \mathrm{ml}$ to $300 \mathrm{pg} / \mathrm{ml}$ ).

\section{METHODS}

The study was designed as an open label randomized controlled trial (clinical trials registration number (Clinical Trials.gov ID NCT00939718). Patients were enrolled from outpatient clinics of the department of Psychiatry at Aga Khan University Hospital, Karachi Pakistan from December 2009 - June 2010. Depression was defined as Patients scoring $\geq 16$ on the 20 -item Hamilton Rating Scale for Depression-Urdu version (HAM-D) [23,24].

Low normal B12 level was defined as B12 level ranging between 190 and $300 \mathrm{pg} / \mathrm{ml}$. Patients with B12 deficiency (level below190 pg/ml) were not enrolled due to ethical reasons (patients with established B12 deficiency must be treated and not subjected to randomization).

\section{Ethics Statement}

A study protocol was approved by the Ethics Review Committee of Aga Khan University hospital. All patients signed an informed consent form.

\section{Randomization and Masking}

Patients with depression and low normal B12 levels were randomized by a computer program into the control arm (antidepressants only) or treatment arm (antidepressants and injectable vitamin B12 supplementation). The randomization was carried out using a Microsoft Excel spreadsheet using the function "RAND" to generate uniform random numbers.

The randomized assignment was determined by partitioning the range of the random number. Randomization was not concealed for investigators. Antidepressants that were prescribed included either a tricyclic antidepressant (TCA) with a dose equivalent of imipramine $100 \mathrm{mg}$ to $250 \mathrm{mg}$ / day and the SSRIs dose equivalent of Fluoxetine $20-40 \mathrm{mg} /$ day. Patients with concurrent unstable medical illness, history of manic episodes or psychotic illness, psychotic symptoms in a depressive episode, concurrent substance misuse and patients with suicidal ideation were excluded. Participants in the control arm only received the antidepressants. Those in the treatment arm received B12 intramuscular injectable as 1000 mcg every week during the 6 weeks in addition to the antidepressants.

Any adverse effects of oral medications and injections (B12) were monitored and documented on an adverse effects sheet in the patient folder.

A decline in HAM-D score of $20 \%$ or more from the baseline, indicating an improvement in depression, was defined as the primary outcome. The total change in HAM-D score from baseline to follow up was defined as a secondary outcome. Additional secondary outcomes included the follow up HAM-D score and a reduction in HAM-D score of 50

or more from the baseline. Follow up of the HAM-D score was recorded during 12 weeks by a research officer who was unaware of the patient's randomization.

\section{Statistical Considerations}

A total of 248 patients equally allocated to the two groups provides at least 90 percent power, with a 5 percent type I error rate, using a two-sided hypothesis test. This calculation assumes an anticipated difference in the response rate of 20 percent between the two groups with a 30 percent response rate in the SSRI group and at least a 50 percent response rate in the combination treatment group. Considering a 15 percent dropout rate in each group, an additional sample of 44 patients equally divided into the two groups would be needed to yield a total required sample size of 292 patients. Therefore, the target sample size for each arm was 146.

Descriptive statistics were prepared for all characteristics including means and standard deviations for continuous measures and frequencies and percentages for categorical measures. Chi-square or Fisher's exact tests were used to compare categorical baseline characteristics. Two-sample $t$ tests were used to compare continuous baseline characteristics. Chi-square tests were used to examine the association between treatment and the $20 \%$ and $50 \%$ reductions in HAM-D outcomes. These analyses were extended to logistic regression to adjust for the baseline HAM-D score. Twosample $\mathrm{t}$ tests were used to examine the association between treatment and the follow up HAM-D score or change in HAM-D score from baseline to follow up. These analyses were extended to analysis of covariance to adjust for the baseline HAM-D score. All hypotheses tests were two-sided and p-values less than 0.05 were considered statistically significant. Analyses were conducted using SAS Version 9.2 (SAS Institute, Inc., Cary, NC, USA).

\section{Results}

A total of 199 depressed patients were screened for the B12 levels. Vitamin B12 deficiency was present in $44(22 \%)$ patients; 73 (36\%) had low normal B12 levels and 82 (42\%) had normal B12 levels. Patients with low B12 levels were given the prescriptions for B12 replacement therapy in addition to the antidepressants. These patients were not randomized. Out of 73 patients with low normal B12 levels 34 $(47 \%)$ were randomized to the treatment group while 39 (53\%) were randomized to the control arm. There were no significant differences between the two groups at baseline except for the higher depression scores in the treatment group (Table 1). No adverse effects or complications were noted in either group. For the primary outcome of $20 \%$ reduction in HAM-D score, significantly more subjects from the treatment group showed a $20 \%$ reduction unadjusted for baseline HAM-D score $(100 \%$ vs. $69 \%$; $p<0.001)$. Examining a50\% reduction from baseline, this effect remained significant $(44 \%$ vs. $5 \%$; p < 0.001$)$. We also adjusted the analyses of reduction for the baseline HAM-D score and our findings remained significant (Table 2). Additionally, the change in HAM-D score was significantly greater for the 
Table. 1. Baseline Characteristics Shown as Mean \pm Standard Deviation or Frequency (Percent)

\begin{tabular}{|c|c|c|c|}
\hline & Treatment (n=34) & Control $(n=39)$ & p-value \\
\hline Age, in years & $37.68 \pm 13.38$ & $36.56 \pm 12.28$ & 0.71 \\
\hline Vitamin B12 level & $238.49 \pm 33.21$ & $245.16 \pm 27.82$ & 0.36 \\
\hline \multicolumn{4}{|l|}{ Gender } \\
\hline Female & $16(47.1)$ & $19(48.7)$ & \\
\hline \multicolumn{4}{|l|}{ Marital status } \\
\hline Single never married & $9(26.5)$ & $13(33.3)$ & 0.84 \\
\hline Married & $21(61.8)$ & $21(53.9)$ & \\
\hline
\end{tabular}

treatment group, unadjusted and adjusted for the baseline HAM-D score (Table 2).

\section{DISCUSSION}

Coexistence of depression and vitamin B12 deficiency are not uncommon. These patients are routinely treated with SSRI and B12 supplementation, however it is not well established whether the people with low normal B12 and cooccurring depression should also receive B12 supplementation. Our study tried to address this issue. Despite not attaining the targeted sample size, the findings appear significant. Vitamin B12 deficiency was present in 22\% of our depressed population. This frequency is high in a non-vegetarian, relatively young, middle to upper income population of our patients. A recent study of healthy adults from Karachi showed a population based prevalence of vitamin B12 deficiency (less than $200 \mathrm{pg} / \mathrm{ml}$ ) in $9.74 \%$ people [25]. These findings indicate a substantial co-morbidity of B12 deficiency (22\%) in our depressed patient population.

To our knowledge, this is the first randomized control trial in Pakistani population which has looked at clinically depressed patients with low B12 levels. We did not randomize B12 deficient patients due to ethical reasons. Low normal B12 levels were present in $36 \%$ of patients. These patients are often not treated with B12 supplementation. The findings of our study clearly indicate that these patients demonstrated significant improvement with B12 supplementation in addi- tion to SSRI as compared to the control group even after adjustment for baseline HAM-D score. We think that these patients represent sub group within the clinically depressed population and a supplementation with B12 along with the conventional antidepressants may be a useful strategy in the treatment of depression in such cases.

We did not give sham injections to the control group. A placebo response due to injections may be responsible for the significant improvement in the treatment group. We also did not look at differences in response at specific doses of antidepressants nor did we stratify our groups on the basis of type of antidepressants prescribed. We also could not reach our sample size because we were not able to get another extension in our grant. Due to financial constraints we were not able to obtain final B12 levels. These are some of the limitations of our study. However, these findings have important clinical implications. B12 deficiency and low normal B12 levels are common and may be associated with depression and the inadequate response to antidepressant treatment in patients with depression. Vitamin B12 supplementation with antidepressants has significantly improved depressive symptoms in our group. Larger, multi-center studies are required to extend and replicate our findings.

\section{CONFLICT OF INTEREST}

Authors have no conflicts of interests to declare

Table 2. Outcomes for Follow Up After 3 Months Shown as Mean \pm Standard Deviation or Frequency (Percent)

\begin{tabular}{|l|c|c|c|c|}
\hline & Treatment (n=34) & Control (n=39) & Unadjusted P-value & Adjusted* P-value \\
\hline \hline $20 \%$ reduction in HAM-D score & $34(100)$ & $27(69.2)$ & $<0.001$ & 0.001 \\
\hline $50 \%$ reduction in HAM-D score & $15(44.1)$ & $2(5.1)$ & $<0.001$ & $<0.001$ \\
\hline Follow up HAM-D score & $12.12 \pm 5.12$ & $14.38 \pm 4.73$ & $<053$ & $<0.001$ \\
\hline Change in HAM-D score & $11.09 \pm 4.58$ & $5.00 \pm 3.38$ & $<0.001$ & $<0.001$ \\
\hline
\end{tabular}




\section{CONSORT}

2010 Flow

Diagram

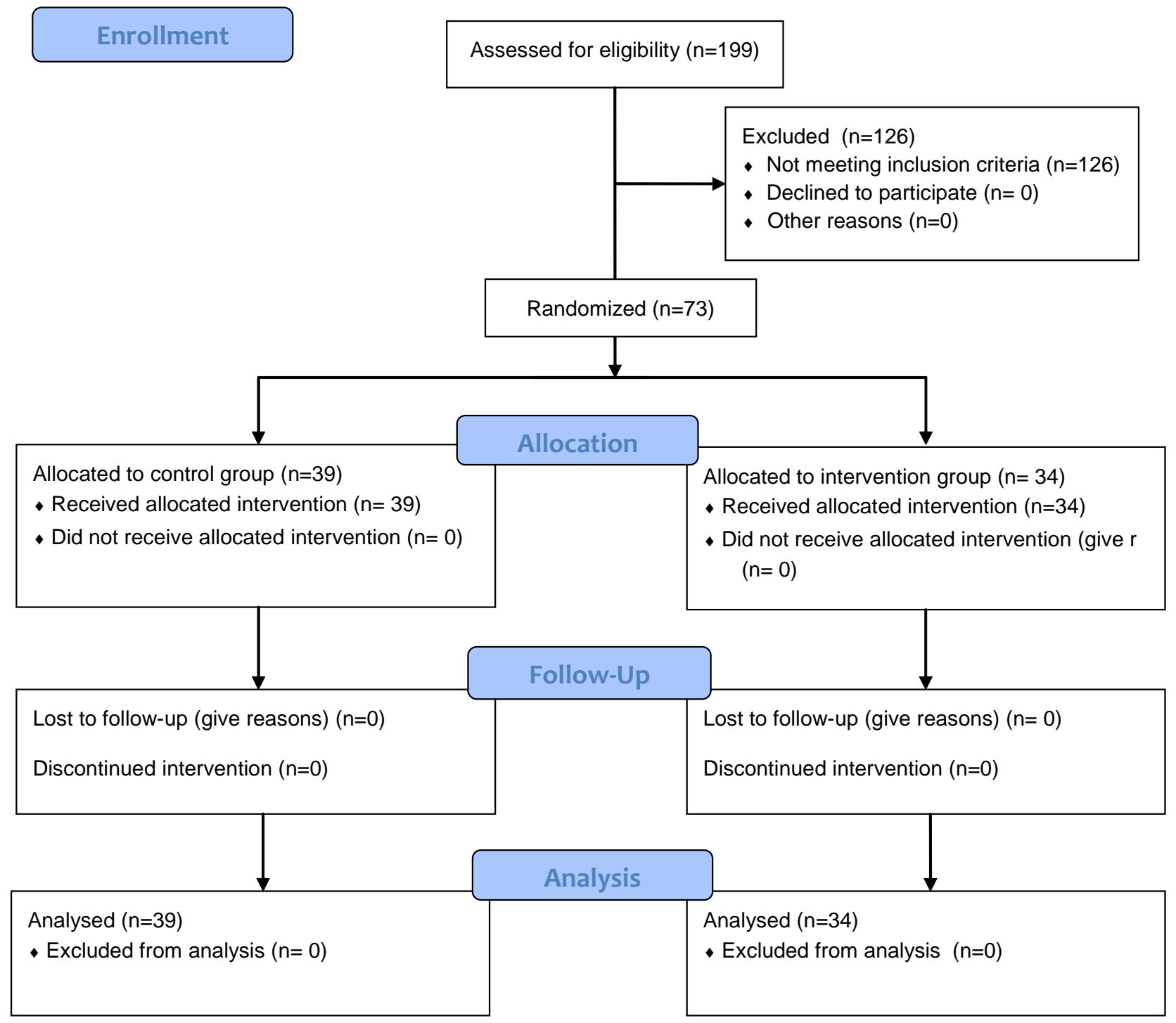

*Adjusted for baseline difference in HAMD scores in the two groups

\section{ACKNOWLEDGEMENT}

Authors would like to acknowledge Michele Shaffer PhD from School of Public Health, Penn State University for her valuable input in statistical analysis and manuscript revision.

\section{FUNDING}

Study funding was supported by University research council grant of Aga Khan University (G\&C code; 70210)

\section{REFERENCES}

[1] Murray C, Lopez A. The global burden of diseases: a comprehensive assessment of Mortality and disability from diseases, injuries and risk factors in 1990 and projected to 2020. Boston: Harvard School of Public Health, WHO and World Bank 1996.
[2] Mirza I, Jenkins R. Risk factors, prevalence, and treatment of anxiety and depressive disorders in Pakistan: systematic review. BMJ 2004; 328; 794-8

[3] American Psychiatric Association; Practice Guideline for the Treatment of Patients With Major Depressive Disorder, $3^{\text {rd }}$ ed. DOI10.1176/appi.books.9780890423387.654001

[4] Thase ME, Entsuah AR, Rudolph RL. Remission rates during treatment with Venlafaxine or selective serotonin reuptake inhibitors. Br J Psychiatry 2001;178: 234 -41

[5] Puech A, Montgomery SA, Prost JF, Solles A, Briley M. Milnacipran;a new serotonin and Noradrenaline reuptake inhibitor: an overview of its antidepressant activity and clinical tolerability. Int Clin Psychopharmacol 1997; 12(2):99-108.

[6] Antai-Otong, D . Monotherapy Antidepressant: A Thing of the Past Implications for the Treatment of Major Depressive Disorder Perspect Psych Care 2007: 43(3): 142-5

[7] Shah RA, Qureshi MB, Khan AA. Magnitude of vitamin A deficiency in poor communities of the four selected districts of Punjab 
using - (rapid assessment technique). Ann King Edward Med Coll 2005; 11(3): 314-8

[8] Khan RM, Iqbal P. Deficiency of vitamin C in South Asia. Pak J Med Sci 2006; 22(3): 347-55

[9] Hashim H, Tahir F. Frequency of vitamin B12 and folic acid deficiencies among patients with megaloblastic anemia. Ann Pak Inst Med Sci 2006; 2(3): 192-4

[10] Yajnik CS, Deshpande SS, Lubree HG, et al. Vitamin B12 deficiency and hyperhomocysteinemia in rural and urban Indians. $\mathbf{J}$ Assoc Physicians India 2006; 54: 775-82

[11] Fakhrzadeh H, Ghotbi S, Pourebrahim R, et al. Total plasma homocysteine, folate, and vitamin b12 status in healthy Iranian adults: the Tehran homocysteine survey (2003-2004)/a cross - sectional population based study. BMC Public Health 2006; 6: 29

[12] Lim HS, Heo YR. Plasma total homocysteine, folate, and vitamin B12 status in Korean adults. J Nutr Sci Vitaminol (Tokyo) 2002; 48(4): $290-7$

[13] Refsum H, Yajnik CS, Gadkari M, et al. Hyperhomocysteinemia and elevated methylmalonic acid indicate a high prevalence of cobalamin deficiency in Asian Indians. Am J Clin Nut 2001; 74(2): 233-241

[14] Robert C, Brown DL. Vitamin B12 Deficiency. Am Fam Physician 2003; 67: 979-86.

[15] Hintikka J, Tolmunen T, Tanskanen A, Viinamäki H. High vitamin B12 level and good treatment outcome may be associated in major depressive disorder. BMC Psychiatry 2003; 3:17-22

[16] Tiemeier H, van Tuijl HR, Hofman A, Meijer J, Kiliaan AJ, Breteler MM. Vitamin B12, folate, and homocysteine in depression: the Rotterdam Study. Am J Psychiatry 2002; 159(12): 209901
[17] Sachdev PS, Parslow RA, Lux O, et al. Relationship of homocysteine, folic acid and vitamin B12 with depression in a middle- aged community sample. Psychol Med. 2005; 35(4): 529-38.

[18] Bottiglieri T, Laundy M, Crellin R, Toone BK, Carney MW, Reynolds EH: Homocysteine, folate, methylation, and monoamine metabolism in depression. J Neurol Neurosurg Psychiatry 2000: 69; 228-232

[19] Alpert JE, Mischoulon D. "Folinic acid (Leucovorin) as an adjunctive treatment for SSRI-refractory depression. Ann Clin Psychiatry 2002; 14(1):33-8

[20] Fava M, Borus JS. "Folate, vitamin B12 and homocysteine in major depressive disorder". Am J Psychiatry 1997; 154(3): 426-8

[21] Papakostas GI, Peterson T. "Serum folate, vitamin B12, and homocysteine in major depressive disorder, Part I: predictors of clinical response in fluoxetine-resistant depression". J Clin Psychiatry 2004; 65(8): 1090-5

[22] Papakostas GI, Peterson T. Serum folate, vitamin B12, and homocysteine in major depressive disorder, Part II: predictors of clinical response in fluoxetine-resistant depression. J Clin Psychiatry 2004; 65(8): 1096-8

[23] Hamilton M. A rating scale for depression. J Neurol Neurosurg Psychiatry 1960; 23: 56-62

[24] Chaudhry HR, Qureshi Z, Tareen IA, Yazdani I. Efficacy and tolerability of Paroxetine $20 \mathrm{mg}$ daily in the treatment of depression and depression associated anxiety. J Pak Med Assoc. 2002; 52(11): 518-25

[25] Yakub M, Iqbal MP, Kakepoto GN, et al. High prevalence of mild hyperhomocystienemia and folate, B12 and B6 deficiencies in an urban population in Karachi, Pakistan. Pak J Med Sci 2010; 26 (4): 923-929.

(C) Syed et al.; Licensee Bentham Open.

This is an open access article licensed under the terms of the Creative Commons Attribution Non-Commercial License (http://creativecommons.org/licenses/by-nc/3.0/) which permits unrestricted, non-commercial use, distribution and reproduction in any medium, provided the work is properly cited. 